areas of interest in both the fundus photos and neuro images for the sake of both exactitude and speed of comprehension.

In general, this is a concise and easily accessible quick reference, well illustrated, covering the spectrum of optic disorders, which should serve as a useful adjunct in the library of the practising ophthalmologist. It is not meant to supplant the more detailed neuro-ophthalmic texts in existence and does list a short but germane bibliography. While Dr. Spoor does emphasize certain personal preferences of management, he recognizes the controversy surrounding these in other areas.

All in all, I think that this is a useful book.

\section{J.R. Buncic,}

Toronto, Ontario

SURGERY OF CRANIAL BASE TUMORS. 1993. Edited by Laligan N. Sekhar and Ivo P. Janecka. Published by Raven Press. 870 pages. $\$ 270.00 \mathrm{Cdn}$.

This multi-author text covers the complete gamut of skull base surgery. Written by some of the surgeons who pioneered and refined modern day skull base surgery, it is the "state of the art" today. The multi-authorship reflects the need for a multidisciplinary team approach to these tumors.

The book proceeds in an orderly fashion from instrumentation, diagnostic investigations, anaesthesia, neuro-physiologic monitoring to anatomy, operative techniques of the various surgical approaches, treatment of specific tumors, to rehabilitation and complications.

For those of us who have visual memories, perhaps the best part of this text are the illustrations, the majority of which are in color. These vary from anatomic specimens, clinical and intraoperative photographs, to superb drawings. These serve to complement a concisely written text.

This book is a must for anyone involved in the management of these difficult tumors.

M.R. MacRae, Calgary, Alberta

TOURETTE SYNDROME: GENETICS, NEUROBIOLOGY, AND TREATMENT. Advances in Neurology, Volume 58. 1992. Edited by Thomas N. Chase, Arnold J. Friedhoff, Donald J. Cohen. Published by Raven Press. 399 pages. \$138.00 Cdn.

This volume is based on an interdisciplinary symposium on Tourette syndrome (TS) held in 1991 and describes advances in the understanding of TS and associated conditions which have occurred over the last decade. As stated in the preface, there is considerable debate within the field and the editors have allowed the individual authors to argue for their own ideas. This is very much a book about work in progress. The first section deals with the clinical phenomenology of TS and of associated phenomen such as obsessive-compulsive disorder. These chapters are well-written and provide a valuable introduction for the individual who wishes to learn about clinical aspects of the disorder. The broad spectrum of tic subtypes is reviewed in detail with ample clinical description thereof. A later section returns to the issue of associated phenomena, including anxiety disorders and self-injurious behaviour and a useful differential diagnosis of the latter is provided.

Pathophysiology of TS is discussed in sections on neurochemistry/neuropathology, genetics, and neuroimaging. All of the reported studies are preliminary in nature but ample speculation is provided regarding the role of potential underlying abnormalities. Singer reports an inability to demon-strate abnormalities in vitro in D1 or D2 receptors (although with singlepoint analyses of binding), in agreement with PET studies of D2 binding reported by Brooks et al., but in contrast to his own PET studies which report a spectrum of D2 changes, ranging from decrease to very elevated $B_{\max }$ values. Several chapters on genetic studies establish the difficulty at finding the gene responsible for this presumed hereditary disorder. An excellent chapter by David Pauls discusses the underlying issues and points out that a full characterization of the spectrum of the TS phenotype is lacking so that some of these studies may have included inappropriate subjects while others may have erroneously excluded appropriate subjects. An interesting observation, made by McMahon et al., is that "like marries like" - in families with TS, there is an inordinately high number of unrelated but affected spouses.

Both pharmacological and non-pharmacological treatments are reviewed, allbeit briefly. A review of the use of neuroleptics is disappointingly brief but does emphasize that their use should be limited to those in whom tics have led to significant problems. Of more practical value is the discussion of clonidine and clonazepam and that of the psychopharmacology of obsessivecompulsive disorder in TS. The final chapter provides a valuable conclusion, summarizing the current state of the diagnosis, pathophysiology, and management of this puzzling disorder.

This book will be useful as a source of current information for any professional who has a major interest in TS. All neurologists, psychiatrists, and residents in these specialties will benefit from reading the introductory clinical chapters and the concluding remarks.

W.R. Wayne Martin, Edmonton. Alberta

\section{EPILEPTIC SYNDROMES IN INFANCY, CHILDHOOD} AND ADOLESCENCE. SECOND EDITION. Edited by J. Roger, M. Bureau, C. Dravet, F. Dreifuss, A. Perret and P. Wolf. Published by J. Libbey \& Co. 418 pages. $\$ 61.00 \mathrm{Cdn}$.

The first edition of this book arose from the proceedings of a workshop on Childhood Epileptology held in Marseille in 1983. The justification for publishing it sprang from the predominantly European school of emphasis on the delineation of syndromes in epilepsy and the fact that there was no easy access to a collection of this information in one book.

I have the first edition and find it most useful. It does however suffer from the drawbacks of many workshop proceedings: the content is uneven, reflecting the multiple authors of different papers, there are the rather uninformative discussions of some papers, and at times the phrasing is somewhat stilted.

The second edition has overcome most of these problems. The text itself is grouped into sections that describe epileptic syndromes in neonates, in infancy and childhood, in childhood 